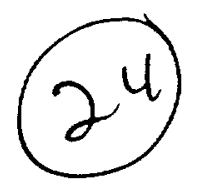

\title{
Scattering properties of needle-like and plate-like ice spheroids with moderate size parameters
}

\author{
Nadia T. Zakharova and Michael I. Mishchenko
}

We use the current advanced version of the $T$-matrix method to compute the optical cross sections, the asymmetry parameter of the phase function, and the scattering matrix elements of ice spheroids with aspect ratios up to 20 and surface-equivalent-sphere size parameters up to 12 . We demonstrate that plate-like and needle-like particles with moderate size parameters possess unique scattering properties: their asymmetry parameters and phase functions are similar to those of surfaceequivalent spheres, whereas all other elements of the scattering matrix are typical of particles much smaller than the wavelength (Rayleigh scatterers). This result may have important implications for optical particle sizing and remote sensing of the terrestrial and planetary atmospheres.

OCIS codes: $010.1110,010.1290,010.1310,010.2940,290.1090,290.1310$

\footnotetext{
N. T. Zakharova (crntz@giss.nasa.gov) is with Science Systems and Applications Inc., 2880 Broadway, New York, NY 10025. M. I. Mishchenko is with the NASA Goddard Institute for Space Studies, 2880 Broadway, New York, NY 10025.
} 



\section{Introduction}

Several recent publications $r$ ave taken advantage of the improved version of the $T$-matrix method $^{1}$ and presented an estensive survey of light-scattering properties of polydisperse, randomly oriented spheroids $w$ th moderate aspect ratios (see, e.g., Refs. 2 and 3 and references therein). However, the results o? Refs. 4-6 give an indication that spheroids with extreme aspect ratios may have scattering prop sties dramatically different from those of moderately aspherical particles. Therefore, in this piper we calculate the optical cross sections, the asymmetry parameter, and the scattering ma rix for randomly oriented ice spheroids with aspect ratios up to 20 and equivalent-sphere size pa ameters up to 12 and compare them with analogous results for surface-equivalent spheres and $s_{I}$ heroids with an aspect ratio of 2 . Since needle-like and platelike particles can be abundant in various natural and artificial environments, we conclude the paper by a discuss on of potential ;ractical implications of our results.

\section{Computations}

We performed calculations for 5 particle shapes: spheres, prolate spheroids with semi-axis ratios $a / b=0.5$ and 0.05 , and oblate spheroids with $a / b=2$ and 20 , where $b$ is the rotation (vertical) semi-axis and $a$ is the horizontal semi-axis of a spheroid. The size of a spheroid was specified in terms of the radius of the surface-equivalent sphere $r_{\mathrm{s}}$ (or, equivalently, the radius of the sphere having the same projected $\operatorname{area}^{7}$ ). To suppress the interference structure in light-scattering patterns (Refs. 2, 3, and 8), the computation results for spheres and spheroids with $a / b=0.5$ and 2 were averaged over a narrow gamma distribution of equivalent-sphere radii given by ${ }^{8}$ 
here. his explanation is worth recalling; it requires a brief digre ssion into th: theory of normal modes.

A tide of frequency $\omega$ has a complex tidal admittance $Z(\omega, I)$ at location $L$ which can be written as an expansion of oceanic normal modes (e.g., Platzman, 1991):

$$
Z(\omega, L)=\sum_{k}\left(1-\omega / \sigma_{k}\right)^{-1} S_{k} H_{k}(L)
$$

According to this prescription each mode $k$ contributes to t\} e tidal admittence an amount depending on three factors: a "frequency factor" involving the mode $;$ complex frequency $\sigma_{k}$, a' "shape factor" depending on the spatial coherence betwern the tidal potential and the mode elevation, and a "location factor" which is simp y the modal amplitude $H_{k}$ at $L$.

Normal modes for the world ocean have been computed by several groux According to calculations by Platzman et al. (1981) there are three modes within ? very near the diurnal band, having periods $23.7,25.7$, and 28.7 nours. The latter mode is primarily an Antarctic Kelvin wave and Pacific half-wave, with hardly any influence in the Atlantic Ocean In contrast, the other two mod ss are primarily Aili.ntic modes. The relevant portion of the 25.7-h mode in shown in Figure 1. It has two amphidromes rotating cyclonically in each basin and larges amplitudes in the Ncrth Atlantic, especially the northeast sector. The 23.7 -h mod is not unlike the 25 ." $-\mathrm{h}$ mode in being largest in the North Atlantic and relativel: small elsewhere; it does, however, have an additional amphidrome in the equatorial Atlantic. See Platzman et al. (1981) and Platzman (1985) for a comprehensive set of mode diagrams.

As Cartwright (1975) pointed out, the 25.7 -h mode is spatially well correlated wi $h$ the third-degree component of the diurnal tidal potential, but not with the secon 1 degree component. The relevant spherical harmonics of t'le potential are (Cartwrig it 
and Tayler, 1971):

$$
\begin{aligned}
Y_{2}^{!}(\theta, \phi) & =\sqrt{ }(5 / 24 \pi) 3 \sin \theta \cos \theta e^{i \phi} \\
Y_{3}^{1}(\theta, \phi) & =\sqrt{ }(7 / 192 \pi) 3 \sin \theta\left(5 \cos ^{2} \theta-1\right) e^{i \phi}
\end{aligned}
$$

for spherical coordinates $(\theta, \phi)$. Both functions are of order 1 , as is appropriate for diurnal tides; they are shown in Figure 2. The $Y_{3}^{1}$ harmonic, like the 25.7-h mode, is symmetric about the equator, and both the harmonic and the mode have local maxima near latitudes $0^{\circ}$ and $\pm 60^{\circ}$. In contrast, the $Y_{2}^{1}$ harmonic is anti-symmetric about the equator, and it cannot be expected to easily excite a symmetric mode. Platzman (1984) confirmed this argument by calculating the correlations: he found the shape factor $S_{k}$ is 0.30 for $Y_{3}^{1}$ but only 0.05 for $Y_{2}^{1}$. (Note that the faster 23.7-h mode is also symmetric, but with an additional amphidrome near the equator it is less well matched to $Y_{3}^{1}$ than is the $25.7-\mathrm{h}$ mode.)

If this story of $M_{1}$ is correct, then one naturally suspects that other third-degree diurnal tides are similarly enhanced in the Northeast Atlantic. It is the purpose of this note to show that they indeed are. In additicn, by studying the third-degree tidal admittances across the diurnal band, one may deduce further characteristics of the normal modes and their responses, namely their periods-to be compared with Platzman's-and their specific dissipation $Q^{-1}$.

Like $M_{1}$ the other third-degree diurnal tides are separated from second-degree tides by only 1 cycle in 8.8 years. The other third-degree tides are also significantly smaller than $M_{1}$, which is itself only a few millimeters. Both facts suggest that very long time series of hourly data are required before reliable estimates can be obtained. The data employed below, from three tide gauges, span at least two complete cycles of the lunar node. i.e. at least 37 years. 


\section{Estimates of degree-3 tides}

Hourly sea level data were analyzed from three stations: Newlyn, England $\left(50^{\circ} 6^{\prime} \mathrm{N}\right.$, $\left.5^{\circ} 33^{\prime} \mathrm{W}\right)$, Lerwick, Scotland $\left(60^{\circ} 6^{\prime} \mathrm{N}, 1^{\circ} 8^{\prime} \mathrm{W}\right)$, and Vigo, Spain $\left(42^{\circ} 14^{\prime} \mathrm{N}, 8^{\circ} 44^{\prime} \mathrm{W}\right)$. The data timespans are: 1960-1997 (Newlyn), 1959-1997 (Lerwick), 1943-1990 (Vigo). The data are of high quality and remarkably complete; the longest gaps are a threeweek period during 1977 at Lerwick and two two-week periods during 1953 at Vigo.

The tidal analyses were performed by least-squares fits to sinusoids of known frequencies. All major constituents, including long-period tides and nonlinear compound tides, were accounted for, although in the tables given here (Tables 2-4) only the relevant third-degree diurnals are listed. For these third-degree diurnals, solutions were attempted for all lines whose potential amplitudes exceed $0.3 \mathrm{~mm}$ in the CartwrightTayler expansion. There are five such constituents spanning the diurnal band between $2 Q_{1}$ and $J_{1}, 2 Q_{1}$ is more than 10 times smaller than $M_{1}$, but it is included because of its importance at the edge of the band. The five constituents are separated in frequency by one cycle/month, with a single gap where one normally expects the $K_{1}$ constituent (its largest third-degree line is only $0.22 \mathrm{~mm}$, even smaller than $2 \mathrm{Q}_{1}$ ).

Because of the extraordinarily long lengths of the time series, relevant nodal side lines in constituents can be included as separate estimates, independent of the main lines. For the third-degree diurnals, nodal lines above the $0.3 \mathrm{~mm}$ cutoff exist for constituents $Q_{1}, M_{1}$, and $J_{1}$. The nodal lines of $Q_{2}$ and $J_{1}$ appear to yield genuine third-degree estimates, but the nodal lines of $\mathrm{M}_{1}$ are problematic: one of them clearly cannot be separated from second-degree lines, since (see Table 1) lines 155.565 and 155.645 are of almost identical frequencies, and the other line may be affected by nodal modulations in a weak nonlinear interaction between $L_{2}$ and $K_{1}$ (equivalent to a line at 155.465). Only nodal lines from $\mathrm{Q}_{1}$ and $\mathrm{J}_{1}$ are included in Tables 2-4.

The tables give amplitude and Greenwich phase lag and the magnitude of the 
admittance $Z$, defined in this context as the ratio of the (complex) elevation to the normalized potential. All three quantities include standard error estimates, based on the diagonal elements of the least-squares covariance matrix. The admittance errors for $2 Q_{1}$ are understandably largest owing to its small amplitude in the astronomical potential. The given error estimates appear realistic according to two tests: (1) The original time series were partitioned into year-long segments and the statistical variability of the yearly estimates was compared with the tabulated standard errors (allowing for a $\sqrt{ } n$ factor for $n$ yearly estimates); this test, of course, can only be performed for major second-degree constituents, but the agreement in these terms appeared satisfactory. (2) The error estimates appear to account adequately for the small differences in $|Z|$ and Greenwich phase lag between the $\mathrm{Q}_{1}$ and $\mathrm{J}_{1}$ nodal lines and their main lines, in keeping with a smooth oceanic admittance.

The tidal estimates for $M_{1}$ at Newlyn and Lerwick agree well with those obtained by Cartwright (1975) for these same two stations, and station Vigo similarly shows an enhanced $M_{1}$ admittance. In fact, all three stations display a strong enhancement across the entire diurnal band. This lends considerable weight to Cartwright's original explanation of a resonance between the tidal potential and a single operating normal mode (of $Q$ not too high). Moreover, all five constituents show phase lags increasing towards the north, consistent with the normal mode phases seen in Figure 1. Both the enhanced amplitudes and the consistent phases must be counted as yet further evidence for the existence of oceanic normal modes, supplementing various other evidence discussed by Platzman (1991).

At all three stations, the admittance at $J_{1}$ is comparable to or slightly larger than that at $M_{1}$. More interestingly, all three stations show admittance amplitudes increasing as the frequency decreases away from $\mathrm{M}_{1}$, with $2 \mathrm{Q}_{1}$ having the largest admittance in all cases. If Platzman's single 25.7 - $\mathrm{h}$ mode is the primary contributor to these tides, which is the case according to his tidal synthesis (Platzman 1984, Figure 
10), then the admittances strongly suggest that his period for this mode is too low. A period below that of $Q_{1}$, and possibly below that of $2 Q_{1}$, appears more consistent with the observed tidal admittances: An error of $10-20 \%$ in Platzman's period is not unreasonable given various approximations made in the original calculations (e.g., neglect of self-attraction and crustal loading).

\section{Modal fits}

With the tidal admittance defined across the diurnal band, it is straightforward to use the formalism of Equation (1) to fit hypothetical normal modes (e.g., Platzman 1991). In particular, if one dominant normal mode is presumed to account for the behavior across the band, then we may fit the admittance $Z$ by a simple equation of form

$$
Z(\omega)=(1-\omega / \sigma)^{-1} b
$$

where the modal strength $b$ and frequency $\sigma$ are treated as free parameters. Here $Z, b$, and $\sigma$ are complex, while $\omega$ is real. Of primary interest is the frequency $\sigma$ which determines the modal period $T=2 \pi / \operatorname{Re} \sigma$ and specific dissipation $Q^{-1}=$ $2 \operatorname{Im} \sigma / \operatorname{Re} \sigma$.

Figure 3 shows the result of fitting the tidal admittances at Newlyn, using a weighted, nonlinear, least-squares algorithm. In this case, there are 2 complex parameters to be determined from 5 complex admittances. The resulting frequency corresponds to a modal period of 28.1 hours and $Q$ of 7 . This estimate of $Q$ is about twice the value determined for the diurnal-band Antarctic Kelvin wave but less than the $Q$ determined for the semidiurnal tides of the North Atlantic (e.g. Platzman 1991). It is clear that the fitted period depends sensitively on the admittance at $2 Q_{1}$ : since its amplitude falls well short of the linear trend between $O_{1}$ and $Q_{1}$, the fitted curve is forced downward, causing a peak (and hence estimated period) in the general 
vicinity of $2 Q_{1}$. In this region of the diagram, the fit is satisfactory given the sizes of the error bars. On the other hand, the amplitude fit at $J_{1}$ is clearly poor and suggests the presence of one or more additional modes.

Equation (2) has therefore been augmented with a second oscillator. The resulting fit to the diurnal admittances is shown in Figure 4. The estimated periods in the case are 27.5 and 23.0 hours, somewhat closer to Platzman's 25.7 and 23.7 hours. The corresponding $Q$, around 10 , is higher than the single-mode fit. One should not, however, put too much credence to this augmented fit; we are fitting 4 complex parameters to only 5 complex admittances, and the very good agreement shown in Figure 4 in fact suggests overfitting. Unfortunately, there are no additional tidal frequencies that can be used to constrain a two-mode fit.

Fits to the data at Lerwick and Vigo are quite similar to those at Newlyn. The estimated periods and $Q$ values for all stations are summarized in Table 5. I am disinclined to make a choice as to the "best" estimated period for the fundamental mode, except to say that the available evidence clearly suggests a period longer than Platzman's original 25.7 hours, a period most likely longer than the period of $Q_{1}$, and perhaps in the vicinity of 27 to 29 hours.

Acknowledgments. The tide gauge data employed here were obtained courtesy of the British Oceanographic Data Centre, Bidston Observatory, and the University of Hawaii Sea Level Center. 


\section{References}

Cartwright, D. E., 1975: A subharmonic lunar tide in the seas off Western Europe. Nature, 257, 277-280.

Cartwright, D. E., 1976: Anomalous $\mathrm{M}_{\mathrm{I}}$ tide at Lagos. Nature, 263, 217-218.

Cartwright, D. E., and R. J. Tayler, 1971: New computations of the tide-generating potential. Geophysical Journal of the Royal Astronomical Society, 23, 45-74.

Cartwright, D. E., R. Spencer, and J. M. Vassie, 1986: Some minor but interesting properties of the Atlantic Ocean tides. Proceedings of the 10th Inter'l. Symposium on Farth Tides, R. Vieira, Ed., Consejo Superior de Investigaciones Cientificas, Madrid, 613-620.

Platzman, G. W., 1984: Normal modes of the world ocean. Part IV: Synthesis of diurnal and semidiurnal tides. J. Phys. Oceanogr., 14, 1532-1550.

Platzman, G. W., 1985: Normal modes of the world ocean: maps and tables. University of Chicago, Dept. Geophysical Sciences, $97 \mathrm{pp}$.

Platzman, G. W., 1991: Tidal evidence fror ocean normal modes. Tidal Hydrodynamics, B. B. Parker, Ed., John Wiley and Sons, 13-26.

Platzman, G. W., G. A. Curtis, K. S. Hansen, and R. D. Slater, 1981: Normal modes of the world ocean. Part II: Description of modes in the period range 8 to 80 hours. J. Phys. Oceanogr., 11, 579-603. 


\section{TABLES}

Table 1: $\mathrm{M}_{1}$ tidal amplitudes at Newlyn, England

\begin{tabular}{cccccc}
\hline \hline $\begin{array}{c}\text { Doodson } \\
\text { number }\end{array}$ & Degree & $\begin{array}{c}\text { Frequency } \\
(\% / \mathrm{h})\end{array}$ & $\begin{array}{c}\text { Potential }^{*} \\
(\mathrm{~mm})\end{array}$ & $\begin{array}{c}\text { Equilibrium } \\
(\mathrm{mm})\end{array}$ & $\begin{array}{c}\text { Observed*** }^{* *} \\
(\mathrm{~mm})\end{array}$ \\
\hline 155.445 & 2 & 14.48520 & 1.37 & 0.359 & \\
155.455 & 2 & 14.48741 & 7.41 & 1.943 & \\
155.545 & 3 & 14.48985 & 0.59 & 0.188 & \\
155.555 & 3 & 14.49205 & 3.99 & 1.268 & 4.8 \\
155.565 & 3 & 14.49426 & 0.52 & 0.165 & \\
155.645 & 2 & 14.49449 & 0.59 & 0.155 & \\
155.655 & 2 & 14.49669 & 20.62 & 5.407 & 1.2 \\
155.665 & 2 & 14.49890 & 4.14 & 1.086 & \\
\hline
\end{tabular}

* Potential normalized by acceleration of gravity, extracted from tables of Cartwright and Tayler (1971).

** Equilibrium amplitude equals potential $\times \gamma_{n} Y_{n}^{1}$ where $\gamma_{n}$ accounts for elastic body tide.

*** Sea level amplitudes estimated by Cartwright (1975). 
Table 2: Third-degree diurnal tides at Newlyn, England

\begin{tabular}{ccccccc}
\hline \hline Tide & $\begin{array}{c}\text { Doodson } \\
\text { number }\end{array}$ & $\begin{array}{c}\text { Frequency } \\
(\% / \mathrm{h})\end{array}$ & $\begin{array}{c}\text { Potential }^{*} \\
(\mathrm{~mm})\end{array}$ & $\begin{array}{c}\text { Amplitude } \\
(\mathrm{mm})\end{array}$ & $\begin{array}{c}\text { Phase lag } \\
(\mathrm{deg})\end{array}$ & $|Z|$ \\
\hline $2 \mathrm{Q}_{1}$ & 125.655 & 12.84964 & 0.35 & $0.81 \pm 0.22$ & $205 \pm 16$ & $2.31 \pm 0.62$ \\
& 135.545 & 13.39181 & 0.50 & $1.00 \pm 0.22$ & $247 \pm 12$ & $1.99 \pm 0.43$ \\
$\mathrm{Q}_{1}$ & 135.555 & 13.39402 & 1.28 & $2.82 \pm 0.22$ & $249 \pm 5$ & $2.20 \pm 0.18$ \\
$\mathrm{O}_{1}$ & 145.655 & 13.94768 & 0.65 & $0.93 \pm 0.22$ & $276 \pm 14$ & $1.42 \pm 0.33$ \\
$\mathrm{M}_{1}$ & 155.555 & 14.49205 & 3.99 & $4.67 \pm 0.22$ & $275 \pm 3$ & $1.17 \pm 0.06$ \\
$\mathrm{~J}_{1}$ & 175.555 & 15.59008 & 1.46 & $1.84 \pm 0.22$ & $294 \pm 7$ & $1.26 \pm 0.15$ \\
& 175.565 & 15.59229 & 0.59 & $0.77 \pm 0.22$ & $325 \pm 16$ & $1.30 \pm 0.37$ \\
\hline
\end{tabular}

* Potential normalized by acceleration of gravity, extracted from tables of Cartwright and Tayler (1971).

Table 3: Third-degree diurnal tides at Lerwick, Scotland

\begin{tabular}{ccccccc}
\hline \hline Tide & $\begin{array}{c}\text { Doodson } \\
\text { number }\end{array}$ & $\begin{array}{c}\text { Frequency } \\
(\% / \mathrm{h})\end{array}$ & $\begin{array}{c}\text { Potential } \\
(\mathrm{mm})\end{array}$ & $\begin{array}{c}\text { Amplitude } \\
(\mathrm{mm})\end{array}$ & $\begin{array}{c}\text { Phase lag } \\
(\mathrm{deg})\end{array}$ & $|Z|$ \\
\hline $2 \mathrm{Q}_{1}$ & 125.655 & 12.84964 & 0.35 & $1.35 \pm 0.18$ & $266 \pm 8$ & $3.84 \pm 0.51$ \\
& 135.545 & 13.39181 & 0.50 & $1.66 \pm 0.18$ & $307 \pm 6$ & $3.31 \pm 0.35$ \\
$\mathrm{Q}_{1}$ & 135.555 & 13.39402 & 1.28 & $4.08 \pm 0.18$ & $307 \pm 3$ & $3.18 \pm 0.14$ \\
$\mathrm{O}_{1}$ & 145.655 & 13.94768 & 0.65 & $1.66 \pm 0.18$ & $337 \pm 6$ & $2.54 \pm 0.27$ \\
$\mathrm{M}_{1}$ & 155.555 & 14.49205 & 3.99 & $7.25 \pm 0.18$ & $332 \pm 2$ & $1.82 \pm 0.05$ \\
$\mathrm{~J}_{1}$ & 175.555 & 15.59008 & 1.46 & $2.54 \pm 0.18$ & $354 \pm 4$ & $1.74 \pm 0.12$ \\
& 175.565 & 15.59229 & 0.59 & $1.22 \pm 0.18$ & $15 \pm 9$ & $2.06 \pm 0.30$ \\
\hline
\end{tabular}

Table 4: Third-degree diurnal tides at Vigo, Spain

\begin{tabular}{lcccccc}
\hline \hline Tide & $\begin{array}{c}\text { Doodson } \\
\text { number }\end{array}$ & $\begin{array}{c}\text { Frequency } \\
(\% / \mathrm{h})\end{array}$ & $\begin{array}{c}\text { Potential } \\
(\mathrm{mm})\end{array}$ & $\begin{array}{c}\text { Amplitude } \\
(\mathrm{mm})\end{array}$ & $\begin{array}{c}\text { Phase lag } \\
(\mathrm{deg})\end{array}$ & $|Z|$ \\
\hline $2 \mathrm{Q}_{1}$ & 125.655 & 12.84964 & 0.35 & $1.08 \pm 0.15$ & $179 \pm 8$ & $3.09 \pm 0.42$ \\
& 135.545 & 13.39181 & 0.50 & $1.02 \pm 0.15$ & $229 \pm 9$ & $2.04 \pm 0.30$ \\
$\mathrm{Q}_{1}$ & 135.555 & 13.39402 & 1.28 & $3.11 \pm 0.15$ & $213 \pm 3$ & $2.43 \pm 0.12$ \\
$\mathrm{O}_{1}$ & 145.655 & 13.94768 & 0.65 & $1.29 \pm 0.15$ & $236 \pm 7$ & $1.98 \pm 0.23$ \\
$\mathrm{M}_{1}$ & 155.555 & 14.49205 & 3.99 & $5.48 \pm 0.15$ & $232 \pm 2$ & $1.37 \pm 0.04$ \\
$\mathrm{~J}_{1}$ & 175.555 & 15.59008 & 1.46 & $1.83 \pm 0.15$ & $250 \pm 5$ & $1.25 \pm 0.11$ \\
& 175.565 & 15.59229 & 0.59 & $0.68 \pm 0.15$ & $26 \pm \pm 13$ & $1.15 \pm 0.26$ \\
\hline
\end{tabular}


Table 5: Period and $Q$ from modal fits

\begin{tabular}{lccccccc}
\hline \hline & \multicolumn{2}{c}{ One-mode fits } & & \multicolumn{4}{c}{ Two-mode fits } \\
\cline { 2 - 5 } \cline { 5 - 7 } Station & Period & $Q$ & & Period & $Q$ & Period & $Q$ \\
\hline Newlyn & 28.1 & 6.9 & & 27.5 & 11.4 & 23.0 & 9.2 \\
Lerwick & 28.2 & 6.3 & & 27.6 & 9.2 & 23.4 & 9.9 \\
Vigo & 28.7 & 6.7 & & 27.8 & 6.9 & 24.1 & 11.2 \\
\hline
\end{tabular}

Periods are in hours. 


\section{FIGURE CAPTIONS}

Figure 1: The 25.7-hour mode in the Atlantic Ocean as calculated by Platzman et al. (1981). Solid lines are $60^{\circ}$ phase contours with arrowhead indicating direction of rotation. Dashed lines are amplitude contours, with shading denoting areas with amplitudes exceeding the global rms amplitude. Reproduced from Platzman (1991) with permission.

Figure 2: Contours of the second-degree (top) and third-degree (bottom) spherical harmonic components of the diurnal tidal potential. Solid and dotted lines denote regions of opposite sign.

Figure 3: Amplitude (top) and phase (bottom) of the third-degree diurnal tidal admittance at Newlyn, England, corresponding to constituent estimates tabulated in Table 1. The smooth curve represents a one-oscillator fit to the data. The amplitude peaks at the modal period 28.1 hours. The poor fit to $J_{1}$ presumably indicates the influence of one or more additional modes.

Figure 4: As in Figure 3 but for a two-oscillator fit to the data. 


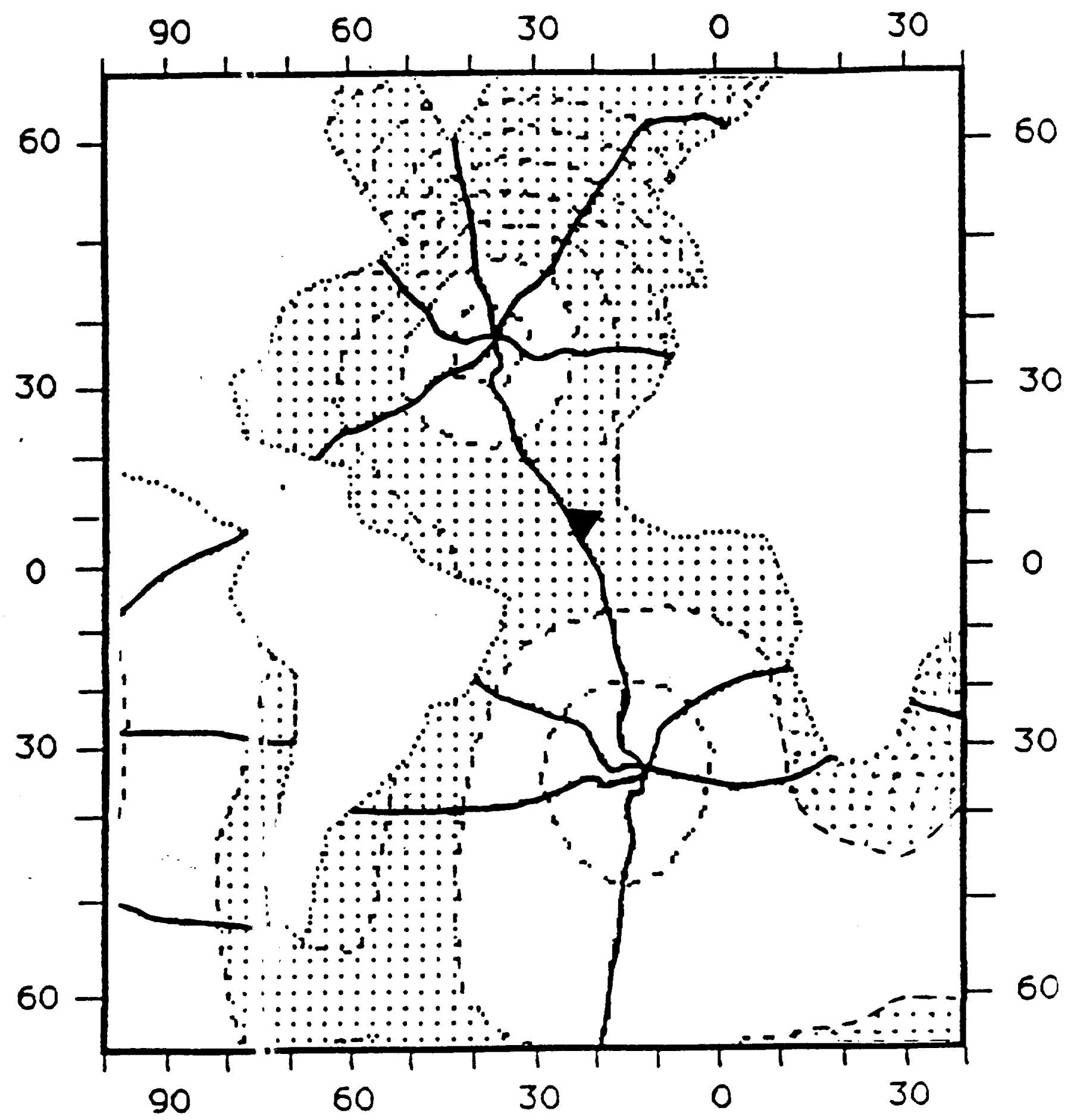

Figure 1: The 5.7-hour mode in the Atlantic Ocean as calculated by Platzman et a. (1981). Sol 1 lines are $60^{\circ}$ phase contours with arrowhead indicating direction of rotation. Dar ied lines are amplitude contours, with shading denoting areas with amplitudes exce ding the global rms amplitude. Reproduced from Platzman (1991) with permission 

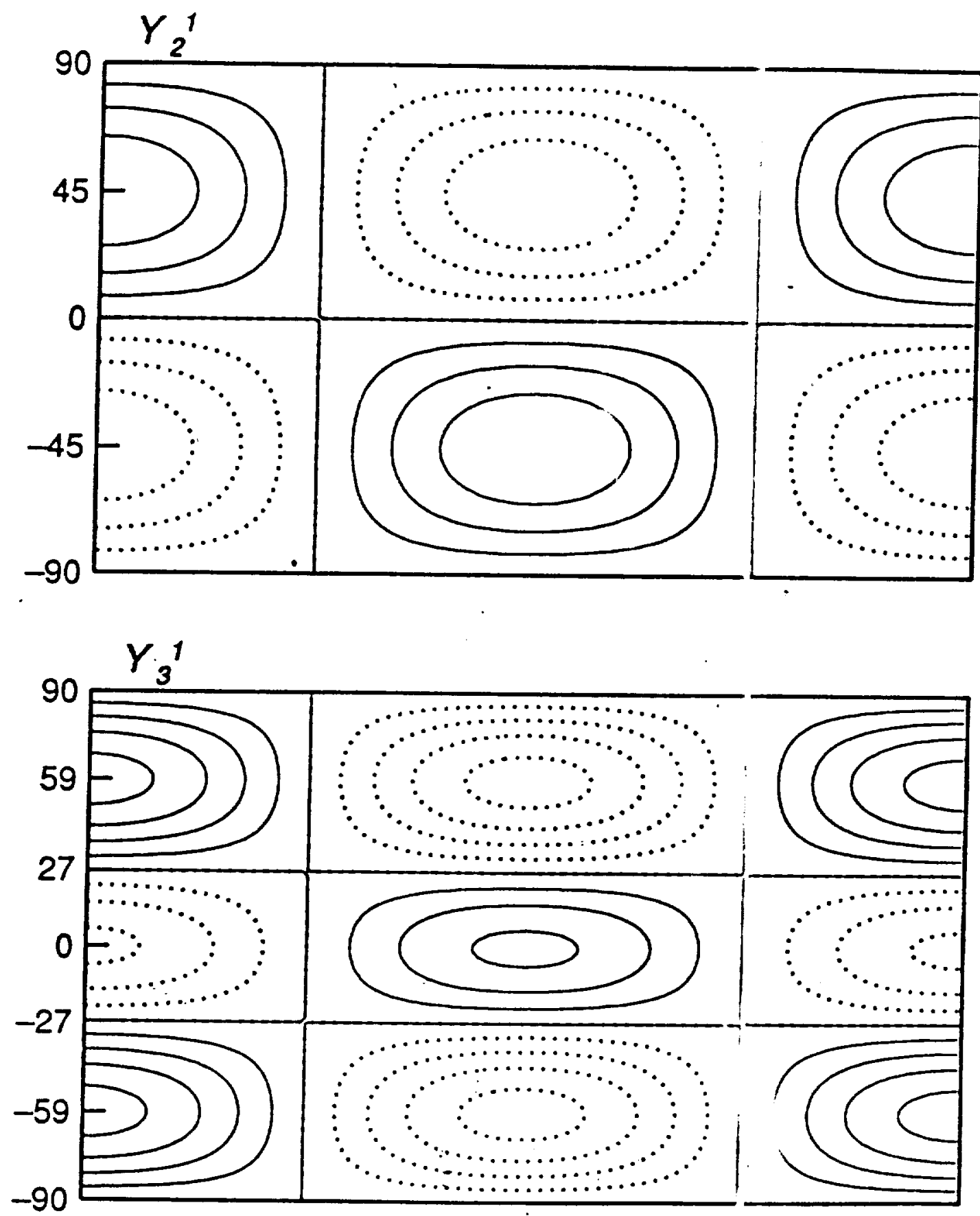

Figure 2: Contours of the second-degree (top) and third-degree (t stt $n$ ) spherical hamonic components of the diurnal tidal potential. Solid and do ted in denote regions of opposite sign. 

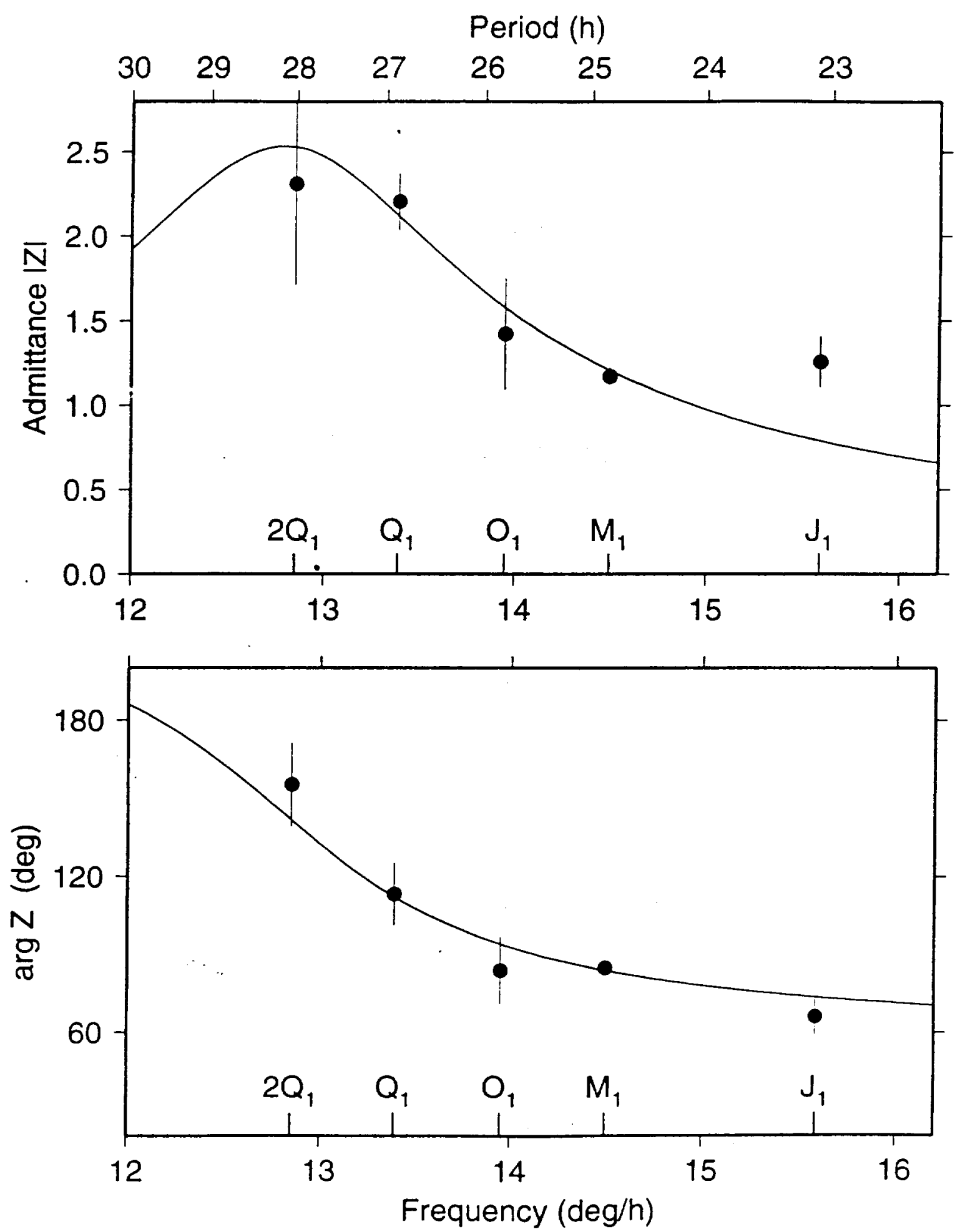

Figure 3: Amplitude (top) and phase (bottom) of the third-degree diurnal tidal admittance at Newlyn, England, corresponding to constituent estimates tabulated in Table 1. The smooth curve represents a ono-oscillator fit to the data. The amplitude peaks at the modal period 28.1 hours. The poor fit to $J_{1}$ presumably indicates the influence of one or more additional modes. 


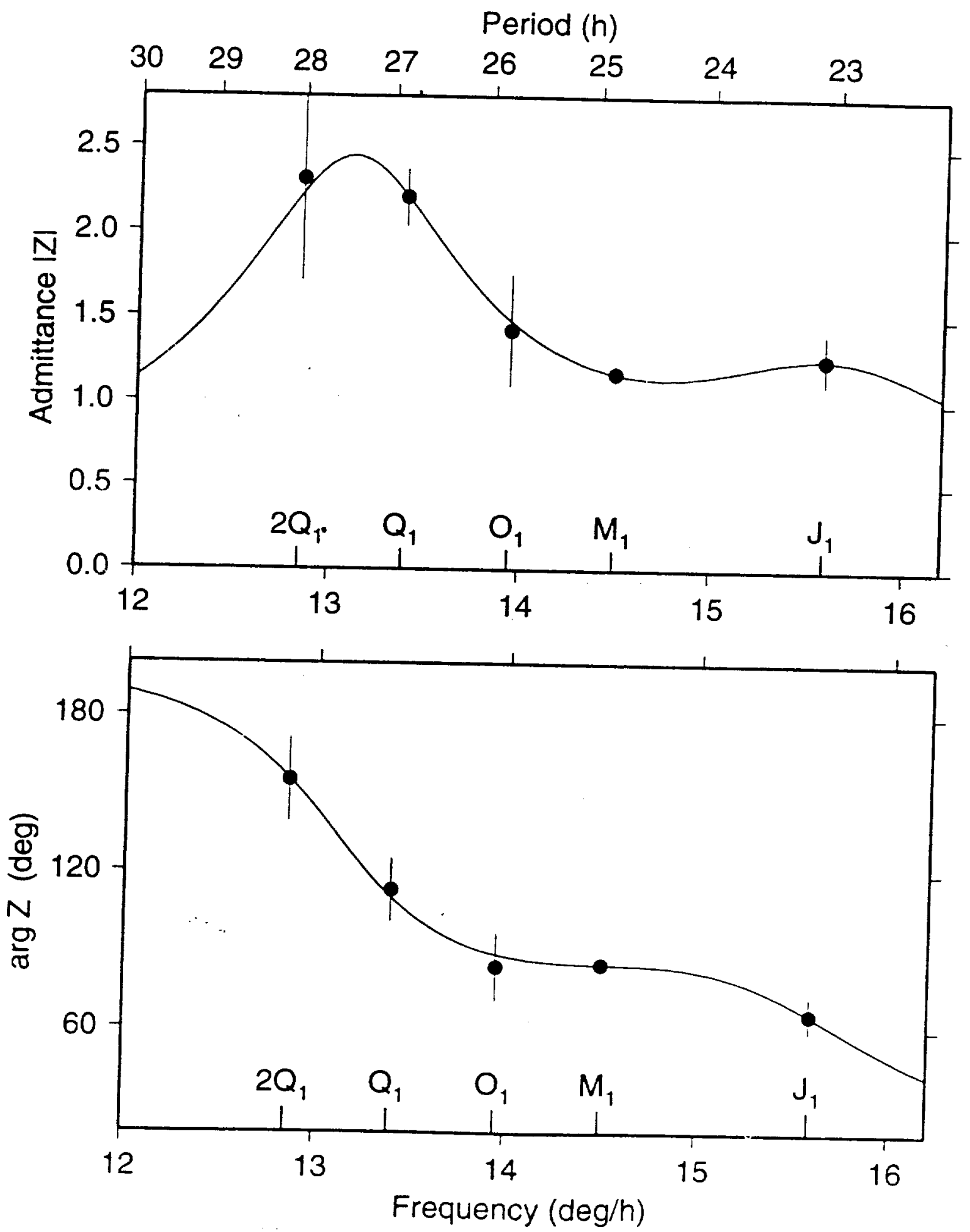

Figure 4: As in Figure 3 but for a two-oscillator fit to the data. 